\title{
Interventionen in Prozessen der Stadt- und Regionalentwicklung. Anmerkungen zum Format Reallabore der Nachhaltigkeit aus planungswissenschaftlicher Sicht
}

\author{
Ulf Hahne \\ Eingegangen: 29. Juli 2020 - Angenommen: 14. April 2021 - Online veröffentlicht: 29. April 2021
}

\begin{abstract}
Zusammenfassung
Reallabore sind ein junges Forschungsformat, mit dem die Nachhaltigkeitswissenschaften zur Lösung realer Problemstellungen vor Ort durch Kollaboration mit Akteuren vor allem auf Stadt- und Regionsebene beitragen möchten. Dies fordert die Planungswissenschaften heraus, ihre eigenen methodischen Ansätze und evidenzbasierten Weiterentwicklungen mit dem neuen Format zu vergleichen. Der Beitrag stellt das neue Forschungs-, Beteiligungs- und Transformationsformat vor und betrachtet explizit seine methodischen Bausteine Realexperiment, Handlungsbezug, Transdisziplinarität und Lernprozesse. Anhand von Beispielen wird auf die vertrackten Probleme komplexer Transformationsprozesse eingegangen, denn Raumentwicklung muss sich stets mit Fragen des Nichtwissens, der Kontingenz und der Überraschungen sowie ortsspezifischen ,Eigenarten“ auseinandersetzen. Die Fehler und Fallstricke, die bei Prozessdesign, Akteurauswahl, Beteiligungsverfahren, Rückkopplungsschleifen, Lernprozessen und Umsetzungsverfahren gemacht werden können, sind gut beschrieben und erlauben es, heute mit deutlich weniger ,Naivität' im Feld der transdisziplinären Forschung und Planung vorzugehen, Beteiligungsformate und Interventionen gezielt und reflektiert zu setzen, Scheitern zum Lernen und Überraschungen für kreative Anregungen zu nutzen.
\end{abstract}

Prof. i.R. Dr. Ulf Hahne, Ökonomie der Stadt- und Regionalentwicklung, Universität Kassel, Gottschalkstraße 22, 34127 Kassel, Deutschland

hahne@uni-kassel.de

(c) (1) ( 2021 Hahne; licensee oekom verlag. This Open Access article is published under a Creative Commons Attribution 4.0 International License.
Schlüsselwörter: Reallabor - transformative Wissenschaft Kontingenz - Nachhaltigkeit - Raumentwicklung

Interventions in processes of urban and regional development. Notes on real-world labs of sustainability from a planning science perspective

\begin{abstract}
Real-world labs have established as a new type of research, participation and transformation format. This challenges the planning sciences to compare their own methodological tools and evidence-based developments with the new approach. This paper introduces the new format and considers its methodological building blocks, i.e. real experiment, action orientation, transdisciplinarity, and learning processes. The contribution will address the wicked problems of complex transformation processes because spatial development deals always with questions of ignorance, contingency, and surprises as well as site-specific idiosyncrasies. The mistakes and pitfalls that can be made in process design, actor selection, participation, feedback loops, learning processes and implementation are well described and make it possible today to proceed with considerably less 'naivety' in the field of transdisciplinary research and planning, to set participation formats and interventions in a targeted and reflected manner, to use failures for learning and surprises for creative suggestions.
\end{abstract}

Keywords: Real-world labs = Transformative research Contingency - Sustainability - Spatial development 


\section{Reallabore als Experimentierräume des Wandels}

In der Nachhaltigkeitsdebatte herrscht einerseits weitgehende internationale Einigkeit über die Ziele und die Umsetzungsebenen (global, national und lokal), andererseits lassen sich Defizite in den Fortschritten der Nachhaltigkeitstransformation deutlich benennen. Daher werden immer wieder Versuche unternommen, mit neuen Instrumenten die Transformation in Richtung Nachhaltigkeit $\mathrm{zu}$ beleben und voranzutreiben. Als ein neuer Vorschlag zur Überwindung der Blockaden und Handlungsdefizite in der Nachhaltigkeitstransformation vor Ort wird seit wenigen Jahren die Einrichtung sogenannter Reallabore von wissenschaftlicher Seite aus vorgeschlagen. Reallabore stellen ein junges Forschungs-, Beteiligungs- und Transformationsformat für die anwendungsbezogene Forschung zur Nachhaltigkeit dar. In einem Reallabor wirken Personen aus Wissenschaft und Praxis, Akteure ${ }^{1}$ sowie Bürgerinnen und Bürgern in einem kollaborativen Forschungs- und Entwicklungsprozess vor Ort zusammen, um gemeinsam Lösungen für praktische oder ,realweltliche“ Fragestellungen und Herausforderungen der Nachhaltigkeitstransformation zu erarbeiten.

Das neue Forschungsformat hat sich im deutschsprachigen Raum in nur kurzer Zeit in Wissenschaft und Förderpraxis etabliert und eine breite wissenschaftliche Literatur hervorgebracht (vgl. Überblicke in Schäpke/Stelzer/ Bergmann et al. 2017; Borner/Kraft 2018; Rose/Wanner/ Hilger 2019). Literatur wie Förderpraxis adressieren dabei häufig die räumlichen Ebenen von Quartier, Stadt oder Region. Der Vorschlag für dieses Format kommt jedoch weder aus der Raumplanungspraxis noch aus den Planungswissenschaften, sondern aus der sozialökologischen Forschung zur Nachhaltigkeit. Daher ist es für die Planungswissenschaften relevant, dieses Format zu betrachten und mit den eigenen Erfahrungen und Erkenntnissen aus der Gestaltung von Transformationsprozessen zu kontrastieren und mögliche neue Impulse für die eigenen Methodiken der Erforschung und Praxis von Beteiligungs- und Aktivierungsprozessen oder der Konfliktbewältigung aufzunehmen. Aus dieser Perspektive heraus werden immer wieder Bezüge zu Prozessen und Erkenntnissen der Stadt- und Regionalentwicklung eingeflochten.

An der Literatur zu Reallaboren fällt zunächst auf, dass

\footnotetext{
${ }^{1}$ Hier werden Praktiker/-innen als Beteiligte aus dem Bereich des Praxiswissens, Akteure als handelnde Personen, Bürgerinnen und Bürger als Adressatinnen/Adressaten oder Mitwirkende mit Lokalwissen, ohne selbst Praktiker/-innen oder Akteure zu sein, bezeichnet.
}

die Erfahrungen aus anderen Wissensbereichen der lokalen Transformation bislang wenig beachtet wurden. So kritisieren Gerhard und Marquardt (2017: 98) die fehlende Berücksichtigung der Ansätze der Citizen Science und Rogga, Zscheischler und Gaasch (2018: 19) die Negierung von Teilen der Transdisziplinaritätsforschung. Ferner wird angemerkt, dass ,bereits existierende Transformationsansätze und Realexperimente (...) bislang kaum aufgegriffen“ (Wagner/Grunwald 2019: 261) wurden. Auch fehle es an einer systematischen Aufarbeitung von Spannungen und Zielkonflikten innerhalb der bereits durchgeführten Reallabore (Engels/Rogge 2018: 28). Was hier thematisiert werden soll, ist die mangelnde Berücksichtigung von Erfahrungen und Erkenntnissen aus Raumplanungspraxis und Planungswissenschaften (De Flander/Hahne/Kegler et al. 2014: 285; Kanning 2018: 7). Denn diese können gerade im Hinblick auf Beteiligungsverfahren und Gestaltung von lokalen Entwicklungsprozessen auf einen breiten Erfahrungsschatz und langjährige Lernprozesse verweisen. Diese Lücke zu reduzieren und damit das Instrument der Reallabore sensitiver für Hindernisse und Fallstricke in transdisziplinären Kollaborations- und Beteiligungsverfahren zu stimmen, ist das Ziel dieses Beitrags. Zudem soll er die Debatte aus der Engführung auf stadtbezogene Interventionen herausführen und Erfahrungen aus Regionalentwicklungsprozessen in suburbanen und ländlichen Regionen einbeziehen.

Der Beitrag befasst sich in Kapitel 2 mit Begriff und Genese des Forschungsformats Reallabore und grenzt es von anderen Formaten der Stadt- und Regionalentwicklung ab. Kapitel 3 behandelt die methodischen Kernelemente von Reallaboren und diskutiert sie aus der Sicht der Raumentwicklung. Kapitel 4 reichert die Diskussion zu Reallaboren mit Lernerfahrungen aus Prozessen der Stadt- und Regionalentwicklung an. Anhand eines Transformationsbeispiels zur Klimaanpassung im Wald, welches alle Elemente eines Reallabors aufweist, werden Fallstricke und Grenzen des Formats aufgeführt. In Kapitel 5 erfolgt eine Übertragung der im Praxisbeispiel gewonnenen Erkenntnisse auf ein theoretisches Modell der gezielten Intervention. Ein Resümee beschließt den Beitrag.

\section{Begriff, Genese und Abgrenzung des Forschungsformats Reallabor}

Der Begriff „Reallabor“ ist a priori nicht beschränkt auf Nachhaltigkeitstransformationen, sondern kann grundsätzlich auch allgemeiner, nämlich als Bezeichnung für zeitlich begrenzte Experimentierräume in technisch-sozialen Systemen verstanden werden. So wird er auch bezüglich der Implementation technischer Innovationen als Testraum für regulative Experimente und Lernprozesse (BMWi 2019: 7) 
verwendet. Bei Reallaboren im weiteren Sinne handelt es sich um analytisch abgrenzbare Interventionen in realweltliche Kontexte. Diese Eingriffe können durch einen exogenen Wandel der Rahmenbedingungen (politische oder wirtschaftliche Krisen, Kriege, Epidemien, Naturkatastrophen) ausgelöst sein oder gezielt (durch Politik, Planung, Förderung, Praxisexperiment) initiiert werden. Systemtheoretisch betrachtet geht es um überraschend eintretende oder gezielt herbeigeführte Störungen eines Systems. Derartige Störungen können das System anregen, sich neu aufzustellen, sie können aber auch systemgefährdend wirken und heftige Gegenreaktionen oder Brüche des Systems bewirken. Bedeutende Merkmale sind die Reichweite (räumlich, zeitlich) und Eingriffstiefe (strukturelle Dimension) von Schock und Interventionen. Ferner können Störungen in realen Systemen, anders als unter klar kontrollierbaren Randbedingungen eines künstlichen Labors, auch zum Systemzusammenbruch führen. Die Geschichte bietet zahlreiche Beispiele für den Wandel sozioökonomischer Systeme durch überraschende Schocks. Die plötzliche Unverfügbarkeit systemrelevanter Elemente - das können Ressourcen, Produktionsfaktoren, Handelspartner oder Institutionen (z. B. Freiheiten) sein - stellt das jeweilige System vor erhebliche Herausforderungen. Der Zusammenbruch des realsozialistischen Experiments in den osteuropäischen Staaten und deren Transformation in unterschiedliche Varianten von demokratischen, marktorientierten bis zu oligarchischen und autokratischen Gesellschaften kann daher auch als Reallabor der Wende interpretiert werden (Ther 2014).

Die hier im Vordergrund stehende Verwendung des Begriffs „Reallabor“ für gezielt geplante Nachhaltigkeitsinterventionen in räumlich abgegrenzte Teilsysteme wird im Folgenden genauer betrachtet. Reallabore werden vom Wissenschaftlichen Beirat der Bundesregierung Globale Umweltveränderungen (WBGU) als ,wissenschaftlich konstruierte Räume einer kollaborativen Nachhaltigkeitsforschung mit Interventionscharakter" definiert (WBGU 2016: 542). Aus dieser knappen Definition lässt sich auf Herkunft, Normativität, Aufbau und Methodik von Reallaboren schlieBen: Der Begriff „Reallabor“ wird vor allem im Kontext der sozialökologischen Forschung zur „Großen Transformation“ (WBGU 2011) verwendet. Normativ wird der Forschung mit der Nachhaltigkeitstransformation ein gesellschaftliches Ziel gesetzt; kollaborativ meint die Einbeziehung von Nicht-Wissenschaftlerinnen und Nicht-Wissenschaftlern, Teams sollen sich transdisziplinär aus Personen aus Wissenschaft und Praxis zusammensetzen. Methodisch schlägt die Definition vor, Interventionen in die gesellschaftliche Praxis zu erarbeiten.

Interventionen dieser Art, also temporäre, gut beobachtete Eingriffe in bestehende Systeme, werden seit Längerem auch in Prozessen der Raumentwicklung eingesetzt. Expe- rimentelle Erprobungen in der Planungspraxis der Stadtund Regionalentwicklung sind dabei kein neues Feld ${ }^{2}$, sondern eine seit Langem gezielt verwendete Methode (Kanning 2018: 7), um bei komplexen Problemen oder bei verkrusteten Strukturen Wandel anzuregen und alternative Lösungen zu erarbeiten. Was unter Experimenten in der Raumplanung verstanden werden kann, verdeutlicht folgendes einfache Beispiel aus der Verkehrsplanung.

Vielfach versucht Stadtpolitik, den Durchgangsverkehr in Stadtquartieren zu reduzieren, um die Lebens- und Aufenthaltsqualität für die Anwohner/-innen zu erhöhen. Welche Straßen sollen aber gesperrt werden? In der Praxis lässt sich häufig beobachten, dass der ausgesperrte Verkehr sich rasch Auswegrouten sucht und so zu ganz anderen, möglicherweise noch weniger erwünschten Schleichverkehren mit Verlagerung der Belastungen führt. Da das Verhalten der Akteure nicht geeignet vorherzusagen ist, nutzt Verkehrsplanung bei derartigen Fragestellungen ein variantenreiches Erprobungsverfahren und setzt temporär mobile Verkehrshindernisse. Diese können an verschiedenen Stellen ausprobiert werden, ehe eine verkehrstechnisch befriedigende und vor Ort akzeptierbare Lösung gefunden ist. Das Experiment produziert mittels seiner Lernschritte neues Wissen. Innerhalb praktischer Experimente können auch institutionelle Experimente, insbesondere zu Beteiligungsverfahren und prozessualer Planung, erfolgen.

Experimente mit verschiedenen Ansätzen und Lösungen können die Vielfalt möglicher Problembearbeitungen vergrößern, Lernprozesse bewirken und die Resilienz in adaptiven Systemen erhöhen (McCormick/Anderberg/ Coenen et al. 2013: 12-13). An Verkehrsexperimenten lassen sich beispielhaft die Effekte technischer Innovationen, die Wirkungen neuer Governance-Modi (Bulkeley/Betsill 2005: 52-55) bis hin zu Fragen der Reduktion von Verkehrsemissionen und Auswirkungen auf das Stadtklima (Evans/ Karvonen 2014: 418-421) studieren. Lernen durch Experimente ist ein nicht nur in der Raumplanung, sondern auch in anderen gesellschaftlichen Kontexten verwendetes Verfahren; der Experimental Turn in den Sozialwissenschaften ist vielfach beschrieben (Callon/Lascoumes/Barthe 2009; Overdevest/Bleicher/Gross 2010) und häufig spezifisch im Stadtbezug etabliert: Der Governance-Ansatz der urbanen Experimente hat sich in den vergangenen Jahrzehnten stark verbreitet und wird in der Literatur als Urban Living

\footnotetext{
${ }^{2}$ Auf die frühere Auffassung der Stadt als extern beobachtbares "Labor oder eine Klinik, wo die menschliche Natur und die sozialen Prozesse bequem und ertragreich studiert werden mögen“ (Park/ Burgess 1984: 46), welche von der Chicagoer Schule der sozialökologischen Stadtforschung in den ersten Jahrzehnten des zwanzigsten Jahrhunderts geprägt wurde, sei verwiesen.
} 
Lab diskutiert (Bulkeley/Castán Broto 2012; Voytenko/ McCormick/Evans et al. 2016: 46; Marvin/Bulkeley/Mai et al. 2018).

Die Debatte um Reallabore als neues Forschungs- und Förderungsformat wurde zunächst vor allem in der deutschen Nachhaltigkeits-Community geführt (Wanner/Hilger/ Westerkowski et al. 2018: 94; Wagner/Grunwald 2019: 261), sie wurde schnell in Forschungsförderung überführt und hat sich daher zunächst wenig um eine Abgrenzung von anderen Formaten bemüht. Reallabore der Nachhaltigkeit grenzen sich von den Urban Living Labs ab, indem sie auf die zwingende Notwendigkeit von Transformationsfortschritten, Transdisziplinarität und expliziten Strategien zum Transfer der Ergebnisse abstellen, die bei Urban Living Labs nicht immer im Vordergrund stehen (Schäpke/Stelzer/ Bergmann et al. 2017: 33, 44). Verfolgt man die Literatur zu Urban Living Labs, so zeigt sich, dass der Nachhaltigkeitsgedanke bislang eher ein Nebenziel in Urban Living Labs ist. So zählt das Handbuch zu Urban Living Labs (McCormick/Hartmann 2017: 6) verschiedene Merkmale wie geographische Einbettung, Experimentieren und Lernen, Beteiligung, Leadership und Evaluation auf, der Aspekt der Nachhaltigkeit taucht dabei jedoch nicht auf. Es wäre an anderer Stelle zu prüfen, inwieweit hier inhaltliche oder nur nomenklatorische Unterschiede bestehen (Gerhard/ Marquardt/West 2017: 5).

Der transformatorische Anspruch in Richtung Nachhaltigkeit, der als Kernmerkmal in der Debatte um Reallabore definiert wird, ist den Ansätzen der Raumplanung und der Stadt- und Regionalentwicklung lange vertraut. Raumplanung befasst sich seit Langem mit Fragen nachhaltiger Entwicklung. Zu denken ist einerseits an informelle Prozesse wie an die lokalen Agenden 21, welche auf Artikel 28 der Rio-Agenda von 1992 beruhen und von verschiedenen Governance-Ebenen initiiert wurden (vgl. Brand/Christ/Heimerl et al. 2001). Andererseits zielt die formelle Raumplanung auf die Reduktion von Raumkonflikten durch institutionalisierte Verfahren. Neben den grundlegenden Normen des $\S 1$ Abs. 5 BauGB $^{3}$ und des $\S 1$ Abs. 2 ROG $^{4}$ wurde auch das praktische Instrumentarium der Raumplanung in Richtung nachhaltiger Raumentwicklung differenziert (ARL 2000). Bei den praktischen Aufgabenstellungen der Stadt- und Regionalentwicklung sind Planer/-innen und Wissenschaftler/ -innen stets mit Akteuren via Kooperation, transdisziplinärer Zusammenarbeit und gegenseitigem Lernen im Aus-

\footnotetext{
3 Baugesetzbuch in der Fassung der Bekanntmachung vom 3. November 2017, das zuletzt durch Artikel 2 des Gesetzes vom 8. August 2020 geändert worden ist.

4 Raumordnungsgesetz vom 22. Dezember 2008, das zuletzt durch Artikel 5 des Gesetzes vom 3. Dezember 2020 geändert worden ist.
}

tausch, die Beteiligung ist dabei ein zentrales und stetig weiterentwickeltes Element von Wandlungsprozessen.

$\mathrm{Zu}$ den Besonderheiten des Formats Reallabore gehört die Rolle der Wissenschaftlerin bzw. des Wissenschaftlers. Sie sollen sich in reale Lebenswelten begeben, um dort gemeinsam mit Praktikerinnen/Praktikern und anderen Akteuren neues Transformations- und Handlungswissen zu erarbeiten, sodass aus der analytisch arbeitenden Transformationsforschung eine handlungsorientierte transformative Forschung wird (,Modus-3-Wissenschaft"; Schneidewind/ Singer-Brodowski 2014: 121). Dies erscheint vielen Wissenschaftsdisziplinen fremd und methodisch unzulässig, da die/der Forschende zugleich als Akteur in den Prozess involviert ist und so seine neutrale Position außerhalb des zu beobachtenden Systems verlässt. In den anwendungsbezogenen Handlungswissenschaften wie der Raumentwicklung sind jedoch derartige Vorgehensweisen nicht neu. Auch die Rollenvielfalt bis hin zu Rollenkonflikten kennen Raumplaner/-innen, wenn sie zugleich als Forschende, als öffentliche Akteure sowie als engagierte Mitwirkende auftreten (Selle 2006: 557; Kanning 2018: 12).

Die Definitionen bieten Anlass, über Wissenschafts-, Gesellschafts- und Systemverständnis zu diskutieren, was hier nicht geschehen soll. Ungeachtet der Definitionsfragen entstanden in der Forschungsförderung die ersten Programme, welche die Begrifflichkeit ohne weitere Diskussion aufnahmen und speziell die räumliche Dimension von Städten und Regionen adressierten. So nahmen ab 2014 verschiedene Förderprogramme des Bundesministeriums für Bildung und Forschung (BMBF) den Begriff Reallabor auf, z.B. die Programme „Nachhaltiges Wirtschaften“, „Zukunftsstadt“, „Kopernikus-Projekte für die Energiewende“, „MobilitätsWerkStatt 2025“. Auf Länderebene war das Land BadenWürttemberg Vorreiter und fördert seit 2015 Reallabore als „,ein neues Modell für die Kooperation von Wissenschaft und Gesellschaft". ${ }^{5}$ Inzwischen finden sich auch bei Einreichungen für Förderprojekte und Förderprogramme verschiedenster Mittelgeber immer mehr Projektskizzen, welche den Begriff in den Titel ihres Antrages schreiben. ${ }^{6}$ Insgesamt scheint sich damit ein neues Forschungsformat zu etablie-

\footnotetext{
5 https://mwk.baden-wuerttemberg.de/de/forschung/ forschungspolitik/wissenschaft-fuer-nachhaltigkeit/ reallabore/ (25.02.2021).

${ }^{6}$ Vgl. z. B. die Gewinnerbeiträge in den Wettbewerben des Bundesministeriums für Wirtschaft und Energie (BMWi) (https://www. bmwi.de/Navigation/DE/Service/Wettbewerbe/ wettbewerbe.html; 25.02.2021) oder Projektanträge im Programm „Nationale Projekte des Städtebaus“ beim Bundesministerium des Innern, für Bau und Heimat (BMI) (https://www.bmi.bund.de/SharedDocs/ pressemitteilungen/DE/2019/04/140-Mio-nat-stadtentwicklung. html (25.02.2021)).
} 
ren. Das folgende Kapitel stellt die methodischen Kernelemente von Reallaboren vor und diskutiert sie aus Sicht der Stadt- und Regionalentwicklung.

\section{Kernelemente von Reallaboren aus der Sicht von Stadt- und Regionalentwicklung}

Grundlegend lassen sich zehn Merkmale von Reallaboren herausfiltern (Schneidewind 2014: 3; De Flander/Hahne/ Kegler et al. 2014: 285; Albiez/Meyer-Soylu/Parodi et al. 2016: 111-114; Schäpke/Stelzer/Bergmann et al. 2017: 4-5; Defila/Di Giulio 2018: 10; Wagner/Grunwald 2019: 261):

- Normatives Ziel: Nachhaltige Transformation

- Intervention in reale Verhältnisse von Stadt und Region (,Zukunftslabore im Kleinen“)

- Realexperimente als methodischer Kern

- Handlungsbezug

- Beschleunigung der Diffusion von Innovationen

- Gemeinsame Anstrengung von Wissenschaftlerinnen/ Wissenschaftlern, Akteuren, Politikerinnen/Politikern und Bürgerinnen/Bürgern (Kollaboration)

- Einbindung lokalen Wissens und lokaler Interessen (Transdisziplinarität)

- Offenheit im Prozess und Ergebnisoffenheit des Prozesses

- Laufender Reflexions- und Lernprozess während der Projektlaufzeit

- Übertragbarkeit und Wissenstransfer

Neben den normativen und deskriptiven Bestandteilen lassen sich drei methodische Kernelemente herausfiltern, die im Folgenden aus der Sicht von Theorie und Praxis der Raumentwicklung diskutiert werden: Realexperimente, Transdisziplinarität und Kollaboration, Lernprozesse und Offenheit.

\subsection{Realexperimente}

Methodischer Kern von Reallaboren sind „Realexperimente" (Schneidewind 2014: 2; De Flander/Hahne/Kegler et al. 2014: 285; Wagner/Grunwald 2015: 27; WBGU 2016: 456; Wanner/Stelzer 2019: 6). Das Reallabor schafft den Rahmen, in welchem Experimente in realweltliche Prozesse mit Interventionen eingreifen. In derartigen Realexperimenten sollen Lösungen für Nachhaltigkeitsherausforderungen entwickelt und getestet werden (Schäpke/Stelzer/Bergmann et al. 2017: 14).

Hier mag zunächst der Begriff des „Experiments“ überraschen (Evans/Karvonen 2014: 416). Das Experiment ist in den Wissenschaften ein eingeführtes Verfahren der ge- planten, nach expliziten Regeln durchgeführten Erkenntnisgewinnung, das in einem abgegrenzten Raum (Labor) mit klar definierten und kontrollierten Randbedingungen stattfindet. Kriterium für die Güte des Experiments ist dann die Reproduzierbarkeit (Janich 2004: 621). Gerade dies kann jedoch für Experimente in sozialen Konstellationen nicht erwartet werden. Hier sind die Vielzahl der Akteure und der auf das Feld wirkenden Einflüsse so komplex, dass die Randbedingungen nicht vollständig erfass- und kontrollierbar sind. Nicht die Reproduzierbarkeit, sondern die Übertragbarkeit und die Lernmöglichkeit können daher Kriterien für die Güte von Realexperimenten in sozialen Gestaltungsprozessen sein.

Das Realexperiment dient dazu, neues Wissen zu erschließen, indem Lösungsansätze für gesellschaftliche Fragestellungen deliberativ verhandelt und experimentell erprobt werden. Das experimentelle Probieren kann ein effektiver Weg beim Handeln unter Unsicherheit sein, um Erfahrungen zu sammeln, Wissen zu erzeugen, es zu korrigieren und Lösungen zu verbessern. „Das Konzept des Realexperiments geht von dem Normalfall aus, dass man relativ viel über das, was man nicht weiß, wissen kann, und dass das Ausprobieren der effektivste Weg ist, sich selbst zu korrigieren und weiterzukommen" (Groß/Hoffmann-Riem/ Krohn 2005: 12).

Das Realexperiment ist damit kein beliebiger Prozess sozialen Lernens, sondern ein strategisches Vorgehen bei gesellschaftlichen Problemstellungen, für die Lösungen erarbeitet werden. Durch Kombination von vorhandenem Wissen mit neu erzeugtem oder neu erhaltenem Wissen, durch rekursives Lernen aus Fehlern und situatives Anpassen der Maßnahmen, manchmal auch der Handlungsziele oder der Akteure, lassen sich neue Ergebnisse und Erkenntnisse erreichen. Überraschungen sind in der Überprüfung der Wissensbestände - sowohl dem der Expertinnen/ Experten als auch dem lokalen Wissen vor Ort - dabei ebenso möglich wie bei der Beteiligung der Akteure und bei der praktischen Umsetzung von Lösungsvorschlägen. Zum Realexperiment gehören daher auch die Kategorien von Offenheit im Prozess (Gerhard/Marquard 2017: 103; Schäpke/Stelzer/Caniglia et al. 2018: 90), geringe Kalkulierbarkeit (Parodi/Waitz/Bachinger 2018: 55) sowie die Spannung zwischen Kontrolle und Kontingenz (Karvonen/van Heur 2014: 388; McCormick/Hartmann 2017: 8; Bulkeley/Marvin/Palgan et al. 2019: 319).

Das Realexperiment unterscheidet sich von der Feldbeobachtung eines neutralen außenstehenden Betrachters durch die aktive Einflussnahme auf den Prozess, die schon durch das bloße Involviertsein in Diskussionsprozesse, Lernkurven, Aktionen, Implementationsverfahren, Evaluationskriterien entsteht. Die Randbedingungen sind dabei meist situ- 
ationsspezifisch und so muss die Übertragbarkeit besonders gesichert werden.

\subsection{Transdisziplinarität und Kollaboration}

Die Forderung nach Transdisziplinarität ist in anwendungsbezogenen Forschungsprojekten kein neues Thema, wenngleich es epistemologisch bislang wenig reflektiert ist (Krohn/Grunwald/Ukowitz 2017: 341). Für Reallabore der Nachhaltigkeit wird Transdisziplinarität explizit als Kollaboration zwischen Universitäten, lokalen Verwaltungen, Unternehmen, Interessengruppen und Bürgerinnen/Bürgern (Engels/Walz 2018: 39) oder akteurbezogen als Zusammenarbeit von wissenschaftlich und nicht wissenschaftlich tätigen Menschen und insbesondere Praxisakteuren (Rose/ Wanner/Hilger 2019: 4) gefordert und dies für alle Phasen des jeweiligen Reallabors: So kann (vielfach: „sollte“) bereits bei der Antragstellung und Problembearbeitung zusammengearbeitet werden, das Vorgehen in Forschung und Praxisexperiment kann im Ko-Design erfolgen, die Projektleitung kann ebenfalls in Kollaboration geschehen, die experimentelle Umsetzung kann in der Ko-Produktion vonstattengehen, am Ende des Projektes kann eine KoEvaluation stehen (Rose/Wanner/Hilger 2019: 6). Dies beschreibt zugleich die Phasen des Projektlebenszyklus von Reallaboren.

Allerdings sind derartige Kooperationen voraussetzungsvoll. Bereits das Erkenntnisinteresse dürfte zwischen Wissenschaft und Akteuren der Praxis auseinanderfallen: Erstere ist an wissenschaftlicher Erkenntnis, letztere sind vornehmlich an einem praktischen Ergebnis interessiert (Di Giulio/Defila/Brückmann 2016: 190). Auch in der Raumplanung wird das Verhältnis von Theorie und Praxis immer wieder - und zwar von beiden Seiten aus - debattiert, zumal eine praxistheoretische Perspektive in der raumwissenschaftlichen Forschung bislang wenig Berücksichtigung gefunden hat (Zimmermann/Feiertag 2020: 42). Häufig bestimmt der Anlass (Ausschreibung, Praxisproblem), wer die Initiative für ein transdisziplinäres Projekt ergreift und damit erste Weichenstellungen schafft hinsichtlich der Ziele, der Auswahl der Kooperationspartner (Institutionen wie Akteure) und des Grunddesigns des Projektes.

\subsection{Lernprozesse und Offenheit}

Ein Kernelement von experimentellen Projekten in Reallaboren ist der gemeinsame Such- und Lernprozess. Dies erweitert die Wissensbestände, verändert den Blick auf den Problemgegenstand, führt zum Test von Varianten und deren Bewertung. In dem Sinne sind Reallabore „Lernarrangements", in denen die Beteiligten die Transformation „lesen“ lernen (,transformative literacy“; Schneidewind/
Singer-Brodowski 2014: 228), wie generell in kollaborativen Beteiligungsprozessen Zugang zu Wissensbeständen vermittelt werden muss, um diese $\mathrm{zu}$ verstehen und weiterentwickeln zu können (Gerhard/Marquardt 2017: 99).

Reflexion und wiederholte rekursive Schleifen sind typisch für das Lernen in adaptiven Systemen (Holling/ Gunderson 2002: 25; Walker/Salt 2006: 74), Unsicherheit und Kontroversen sind fundamentale Bestandteile dieses Lernens (Borner/Kraft 2018: 2). Zum Lernen gehören neben die Sachebene stets auch die Kommunikations- und die Beziehungsebene, um die Konflikte und Blockaden zu erkennen und zu bearbeiten. Daher ist über die Rollen und das Rollenverständnis der Akteure nachzudenken und dies gemeinsam zu reflektieren. In lokalen Entwicklungsprozessen hat es sich dabei bewährt, Expertinnen/Experten, die zugleich als Akteure wirken, nicht auch noch moderieren zu lassen (Selle 2019: 29), dies ist auch für Reallabore zu fordern (Rose/Wanner/Hilger 2019: 15). Schließlich ist auch das rekursive Lernen nicht vor Lock-in-Effekten gefeit: Eingefahrene Verbesserungskreisläufe sind ebenso eine Gefahr für den Erkenntnisprozess wie das Ignorieren externer Wissensquellen. Reallabore erfordern daher immer auch einen beständigen Reflexions- und Lernprozess sowohl hinsichtlich der eigenen Forschungspraxis als auch der gesellschaftlichen Wirkung (Schäpke/Stelzer/Bergmann et al. 2017: 5).

Lernprozesse und Wissensgenerierung beinhalten stets auch das Element der Offenheit: Offenheit im Lernen und für neue Erkenntnisse, die Bereitschaft, Wissenslücken zu bearbeiten und neue zu erkennen. Reallabore verstehen sich daher als offene Prozesse des Wandels. Das gilt nicht für Fragen der Beteiligung (Engels/Rogge 2018: 29; SingerBrodowski/Beecroft/Parodi 2018: 25), sondern auch hinsichtlich der Prozessgestaltung und ihrer didaktischen Anpassung (Schäpke/Stelzer/Caniglia et al. 2018: 92), der Innovationsumgebung (Schäpke/Stelzer/Caniglia et al. 2018: 88; Bulkeley/Marvin/Palgan et al. 2019: 324) und insbesondere für die Ergebnisse (Kullman 2013: 882; Albiez/MeyerSoylu/Parodi et al. 2016: 106). Offene Prozesse lassen sich im Verlauf und in den Ergebnissen nur begrenzt kalkulieren (Parodi/Waitz/Bachinger et al. 2018: 55). Offenheit für Kontingenz, für die Veränderbarkeit der Randbedingungen, für sich öffnende Möglichkeitsräume wie für neu auftretende Hindernisse ist daher ein wesentliches Merkmal von Reallaboren (Krohn/Grunwald/Ukowitz 2017: 343) und Urban Living Labs (McCormick/Hartmann 2017: 8; Bulkeley/ Marvin/Palgan et al. 2019: 320), aber eben auch in Prozessen der Stadt- und Regionalentwicklung, deren Lernerfahrungen im folgenden Kapitel eingebracht werden. 


\section{Lernerfahrungen aus Prozessen der Stadt- und Regionalentwicklung}

Experimente in realen Lebenswelten - und seien es noch so scheinbar triviale Fragen wie die Verkehrsberuhigung im Quartier - müssen sich mit der Vielfalt der Bedingungen, Akteure und Dynamiken auseinandersetzen. Sehr häufig bekommen Planerinnen/Planer, Entwicklerinnen/Entwickler und Reallaborforscher/-innen es mit Problemen zu tun, die sich bislang bekannten, nach erprobten Routinen und Normen ablaufenden Lösungen widersetzen: Sie sind nicht einfach, klar definierbar, verständlich und konsensual lösbar, sondern zeichnen sich durch Komplexität aus, liegen quer $\mathrm{zu}$ fachlichen und sektoralen Bezügen und weisen Unwägbarkeiten aufgrund fehlenden Zukunftswissens auf. Derartige Probleme werden im Unterschied zu den erstgenannten ,zahmen ' Problemen in der Stadtentwicklung nach Rittel und Webber (1973: 160) als ,wicked problems“, als boshafte, widerspenstige, vertrackte Probleme bezeichnet. Sowohl die Reallabore der Nachhaltigkeitsforschung als auch die Handlungsvorschläge der Raumplanung, Stadtund Regionalentwicklung müssen sich stets der Frage stellen, wie Lösungen bei boshaften Problemen erarbeitet werden können und welche sinnerzeugenden Konstrukte dafür eingesetzt werden.

Die Idee der Lösung in inter- und transdisziplinären Konstellationen, der Ko-Produktion von Wissen durch Kollaboration mit Akteuren, durch Akzeptanzschaffung via breiter Beteiligung, in Aufnahme und Förderung zivilgesellschaftlichen Engagements und durch Experimente mögen für Vertreterinnen und Vertreter eines klassischen Wissenschaftsverständnisses zunächst ,,artunspezifische Aufgaben, dem Wissenschaftssystem fremde Kompetenzanforderungen“ (Borner/Kraft 2018: 3) sein, stellen in der Stadtund Regionalentwicklung allerdings einen seit Langem erprobten Weg dar. Dabei haben Akteure der Raumplanung und Raumentwicklung einen mühsamen Lernweg hinter sich, ehe sie bereit waren, das Element der Ko-Kreation von Wissen durch nicht-fachliche Akteure als Prozessbereicherung bei Umsetzungsverhandlungen anzusehen. Mit der Erfahrung aus fünf Jahrzehnten der Entwicklung von Beteiligungsformaten und dem Umgang in der Kollaboration mit Akteuren hat sich auch eine breite Forschung zu erfolgreicher Prozessgestaltung (Bischoff/Selle/Sinning 2005; Gothe 2006), aber auch zu Spannungen und Konflikten in Beteiligungs- und Gestaltungsprozessen etabliert, die kritisch auf Akteurinteressen, Beteiligungsformate und die Wirkung von Beteiligungsverfahren auf politische Entscheidungsprozesse schaut (Selle 2019: 22). In der Literatur zu Reallaboren der Nachhaltigkeit fehlt es bislang an einer solchen systematischen Befassung mit Spannungen und Zielkonflikten (Engels/Rogge 2018: 29), obwohl sie durchaus wahrgenommen werden (Leminen/DeFillippi/ Westerlund 2015: 2; Rose/Wanner/Hilger 2019: 13, 26).

\subsection{Experimente}

Experimentelle Verfahren kennt die jüngere Raumplanung in verschiedenen Formaten. Jede Ausschreibung für Bauprojekte, jede Offenlegung von Bebauungsplänen stellt ein Entdeckungsverfahren dar, bei dem sich zeigt, ob die erwarteten Annahmen zur Attraktivität des Gesamtvorhabens, zur Flächensteuerung oder zur Gestaltung der Ausführung auf eine Nachfrage treffen und von Interessenten und Öffentlichkeit akzeptiert werden. Die Forschung zur Stadt- und Regionalentwicklung wird seit Langem begleitet durch Modellvorhaben, Demonstrationsprojekte und Experimente. In aller Regel dienen Modellvorhaben dem befristeten Ausprobieren neuer Lösungswege mit dem Ziel, diese dann auf weitere Anwendungsfälle zu übertragen oder generell neue Themen zu setzen und damit neue Leitbilder der Entwicklung anzustoßen.

Angeregt durch die Europäische Kampagne für den ländlichen Raum 1987/1988 legte die Europäische Kommission 1991 das Experimentierprogramm LEADER ${ }^{7}$ zur Entwicklung der ländlichen Räume auf. Es forderte die Einrichtung sogenannter lokaler Aktionsgruppen, welche eine gebietsbezogene Entwicklungsstrategie zu entwerfen und ihre Durchführung zu verantworten hatten. Strategie, zielorientierte Entwicklung entlang eines konsensual von den Akteuren der Region erarbeiteten und beschlossenen regionalen Entwicklungskonzeptes, Wissensspillover durch Vernetzung zwischen den Handelnden, dezentrales Regional- und Finanzmanagement in kollektiver Trägerschaft von privaten und öffentlichen Akteuren sind die zentralen Elemente. LEADER gilt inzwischen als ein Musterbeispiel für ein erfolgreich aktivierendes ,Bottum-up-Programm “ und wurde ab der EU-Finanzperiode 2007 als grundlegende Fördermethodik in die Programme zur ländlichen Entwicklung integriert (Hahne 2008: 128).

Diverse Ministerien erproben mittels Modell- und Demonstrationsvorhaben neue Technologien, beispielgebende Kooperationen oder neue Politikmodelle. Das reicht von Einzelmaßnahmen bis zu Modellprogrammen der Nachhaltigkeit (z. B. „Regionen aktiv“ des Bundesministeriums für Ernährung, Landwirtschaft und Verbraucherschutz (BMEL) 2001 bis 2007). Auch die Raumentwicklung in Deutschland kennt seit 1992 die „Modellvorhaben der Raumordnung“ (MORO). Dieses Instrument dient zur Umsetzung eines stärker prozess-, aktions- und projektorientierten Planungs-

7 LEADER = Liaison entre actions de développement de l'économie rurale. 
und Politikverständnisses und sieht die ,praktische Erprobung und Umsetzung innovativer, raumordnerischer Handlungsansätze und Instrumente in Zusammenarbeit zwischen Wissenschaft und Praxis, d. h. mit Akteuren in den Regionen vor Ort" ${ }^{* 8}$ vor. Weiter ist auf das seit 1986 eingesetzte Instrument des ,Experimentellen Wohnungs- und Städtebaus“ (ExWoSt) zu verweisen, mit dem der Bund innovative Planungen und Maßnahmen zu wichtigen städtebauund wohnungspolitischen Themen unterstützt (Jakubowski 2016: 155). Das geht von Demonstrationsbauten (etwa zum Thema energiesparendes Bauen) über lokale Strategien zu Klimaschutz und Anpassung an den Klimawandel bis zu Fragen der Mobilität in Stadtquartieren, um nur einige Beispiele aus bislang über 750 geförderten Experimenten zu geben.

In einem föderalen Staat sind Modellvorhaben auch Ausdruck des Wettbewerbs zwischen und innerhalb der föderalen Ebenen, wobei jeder Initiator mit seinem Modellvorhaben andere von der Richtigkeit des eigenen Modellansatzes überzeugen möchte. Da häufig die Mittel von außerhalb der umsetzenden lokalen oder regionalen Ebene stammen, kann dies auch als ,,persuasiver Interventionismus“ (Wiechmann/ Mörl/Vock 2012: 79) bezeichnet werden, bei dem die Initiative top down entwickelt wird, die Umsetzung allerdings auf die freiwillige Teilnahme der regionalen Akteure angewiesen ist. Zudem gibt es Zweifel, dass ,,auf Freiwilligkeit und Best Practice setzende Modellvorhaben eine effektive Form der Steuerung der Raumentwicklung darstellen“ (Wiechmann/Mörl/Vock 2012: 79) und der breiten Adaption von Nachhaltigkeitsfortschritten einen Schub geben. Schließlich bleibt auch die Frage der Reichweite von Realexperimenten offen, da sie sich in den vorgegebenen Rechtsrahmen sowie in das politische Mehrebenensystem einfügen und hohe Überzeugungskraft entfalten müssen, um dauerhaft implementiert zu werden (Siedentop/Zimmer-Hegmann 2020: 35).

\subsection{Akteure}

Die Literatur zu Reallaboren zeichnet ein sehr positives Bild von Akteuren. Sie sind nachhaltigkeitsmotiviert, kooperationswillig, dialogisch, bereit zur Ko-Produktion, man brauche sie bloß einzuladen (MWFK 2013: 14, 20, 38; WBGU 2016: 20, 419). Doch so einfach ist das nicht: Schon die Frage, wer einzuladen ist, ist nicht trivial. Denn sie differenziert sich rasch in: Welche Akteure sind wann und wie zu beteiligen (Seebacher/Alcántara/Quint 2018: 101)? Dabei wird bereits vorausgesetzt, dass es geeignete und mitwir- kungswillige Akteure gibt. Doch auch dies ist nicht immer gegeben. So stehen in dünn besiedelten ländlichen Regionen häufig nicht einmal quantitativ genügend Akteure zur Verfügung, sodass Beteiligung zu einem mühsamen und zähen, von vielen Zufällen abhängigen Prozess werden kann.

Geht es darum, möglichst breit zu beteiligen und ein ,offenes Labor' zu bieten, um eine große Basis und Vielfalt der Beteiligten zu erreichen, so eignen sich Großgruppenprozesse (z. B. Open Space, Zukunftskonferenz) oder Online-Beteiligungen gut, um vielfältige Ideen zu generieren. Sie sind aber hinsichtlich der Verbindlichkeit der Umsetzung der Ideen häufig zu vage: Es fehlen die fachliche und verwaltungsrechtliche Prüfung der Ideen und die verbindliche Vereinbarung über die Umsetzungsschritte. Werden dann in den weiteren Phasen des Entwicklungsprozesses Ideen nicht wieder aufgegriffen oder wird deren Umsetzung auf spätere Zeitpunkte prolongiert, so sind die Frustration und der Rückzug von Akteuren programmiert. Ein weiterer Nachteil besteht in der grundsätzlichen Schwierigkeit, eher beteiligungsferne soziale Gruppen einzubeziehen (Parodi/Ley/Fokdal et al. 2018: 2).

Oder erscheint es zweckmäßig, nur die für Problembearbeitung und Umsetzung, relevanten“ Akteure (z. B. BMBF 2015: 5; Schäpke/Stelzer/Bergmann et al. 2017: 42) einzubeziehen, um einen zügigen und ergebnisorientierten Prozess zu ermöglichen? Wer gehört dann zu dieser Gruppe und wer kann sich ausgegrenzt fühlen? Möglicherweise führt dies zu einem Zirkelproblem, wenn das ,relevante“ Problem von den für diese Relevanz ausgewählten Akteuren bearbeitet wird (Krohn/Grunwald/Ukowitz 2017: 343) und somit wenig Innovation durch Nischenakteure und spontan entstehende kreative Beiträge entfaltet wird.

Die für den Prozess als relevant identifizierten Akteure sind zudem nicht automatisch auch mitwirkungswillig. Manche machen mit, um ihre organisierten oder individuellen Interessen $\mathrm{zu}$ vertreten, manchmal mit grundlegend obstruktiven Zielen, die nicht offengelegt werden, aber der Machtsicherung oder dem Erhalt des Status quo dienen. Dies ist bei Konfliktthemen nicht selten. Kooperatives $\mathrm{Zu}$ sammenarbeiten ist daher keine Selbstverständlichkeit. Viele Prozesse scheitern auch an der Kommunikation untereinander. Große Bedeutung kommt daher neben dem Prozessund Kommunikationsdesign der Kommunikation selbst bei. Die Moderation sollte dabei durch motivierende, interaktionsfreudige Change Agents (Kristof 2017) mit wertschätzender Haltung und dialogischen Interventionsmethoden auf den Kommunikationsprozess einwirken, um bei den Akteuren die Lust auf Veränderung hochzuhalten.

8 https://www.bbsr.bund.de/BBSR/DE/forschung/programme/ moro/moro-node.html (21.02.2021). 


\subsection{Eigenart}

Schon die Akteurdiskussion zeigte: Reallabore sind in spezifischen sozialen Kontexten unterwegs und müssen sich mit den lokalen Strukturen und Problemen, den Besonderheiten der lebensweltlichen Praktiken und Kommunikationskultur, der lokalen Politikverflechtung bis zur ortsspezifischen ökonomischen Performanz auseinandersetzen. Berking und Löw (2008) lenken den Blick auf die eigensinnigen Strukturen und Praktiken von Städten.

Städte weisen ihre je eigenen Handlungspraktiken - ihre ,Eigenlogik ' - auf, von Ort zu Ort unterscheidbare kulturelle, soziale, politische und ökonomische Strukturen, Praktiken und Denkweisen. Gleich ob es um Muster in der Bürgerbeteiligung (Below/Dopfer 2011: 227), Verhalten in interkommunalen Kooperationen (Seuberlich 2012: 107) oder um Unterschiede lokaler Planungskulturen (Wékel 2011: 215) geht, offenbar wird in „historisch wiederkehrenden Schleifen in einer Stadt immer wieder ähnlich gehandelt“ (Löw 2011: 29). „In manchen Städten herrscht eine nostalgische Grundstimmung, in anderen zählt nur die Erwartung an die Zukunft. In manchen Städten geht alles langsam, in anderen sehr schnell. Bislang völlig unbeachtet bleibt, dass dieses Klima, dieser Rhythmus, den wir als Eigenlogik der Städte bezeichnen, das Arbeiten unterschiedlich erleichtert oder erschwert“ (Löw 2011: 30-31). Um es hinzuzufügen: auch das Arbeiten in Reallaboren.

Auch der Wissenschaftliche Beirat Globale Umweltveränderungen hat in seinem Gutachten zum Beitrag der Städte für eine große Transformation (WBGU 2016) auf die Unterschiede der lokalen Gegebenheiten und Problemstellungen hingewiesen und dafür den Begriff der „Eigenart“ gewählt. Gemeint ist damit in einem deskriptiven Sinn das „Typische einer jeden Stadt, das anhand ihrer sozialräumlichen und gebauten Strukturen, ihrer soziokulturellen Charakteristiken und der lokalen urbanen Praktiken beschrieben werden kann“ (WBGU 2016: 143). Der WBGU plädiert zugleich dafür, den Begriff normativ als „Ziel- oder Orientierungsdimension“ zu verwenden: „Jede Stadtgesellschaft kann und muss (...) auf ihre ,eigene Art' ihren Weg in eine nachhaltige Zukunft suchen“ (WBGU 2016: 9-10). Dies entspricht dem Handlungsaufruf aus der Forschung zur Eigenlogik der Städte: „Über die Eigenlogik der Städte nachzudenken bedeutet (...), maßgeschneiderte Lösungen für diese Stadt und ihre Wirtschaft zu entwickeln“ (Löw 2011: 31).

Reallabore werden folglich in einer spezifischen, unwiederholbaren räumlichen und zeitlichen Konfiguration implementiert („situatedness“; Karvonen/van Heur 2014: 385; Bulkeley/Marvin/Palgan et al. 2019: 322) und müssen sowohl das soziale, kulturelle und politische System als auch das psychische System eines Ortes berücksichtigen. Zum psychischen System gehören die mentalen
Infrastrukturen (Welzer 2011: 11), die einerseits in der übergeordneten Landschaft des ,soziokulturellen Regimes“ (im Sinne der Multilevel-Perspektive von Geels 2011), andererseits in Alltagsroutinen, Gewohnheiten, Wahrnehmungsund Deutungsmustern (Welzer 2011: 30) verankert sind und als Hintergrundüberzeugungen, Werte und Verhaltensweisen in Prozessen wirksam werden. In einem derartigen, ,eigenartigen' lokalen Setting müssen Prozessdesign und Kommunikationsinstrumente gut gewählt sein, um die Bereitschaft der ,eigenständig ' handelnden Akteure zu Veränderungsprozessen anzuregen.

\subsection{Transformationspfade: Scheitern und Lernen - ein Prozessbeispiel zur regionalen Klimaanpassung}

Reallabore sind keine Selbstläufer, die nach einer Blaupause überall ähnlich ablaufen können und dann auch überall ähnlich funktionieren. Auch Scheitern ist immer eine Option. Um das Scheitern wissen aber auch die Akteure innerhalb von Reallaborprozessen. Um ihre Interessen geltend zu machen, spielen sie mit dem taktischen Element der ExitDrohung. Handelt es sich bei den Drohenden um systemrelevante Akteure, etwa Repräsentantinnen/Repräsentanten einer zentralen Gruppe, so wird der Prozess verändert, verzögert oder gar gänzlich neu verhandelt werden müssen. Als Beispiel für derartige Prozesse sei ein Vorhaben aus dem Bereich Klimaanpassung in Waldökosystemen herangezogen.

Der Klimawandel setzt dem Wald durch Klimaerwärmung, verlängerte Vegetationsperioden, Trockenstress und Extremniederschläge zu. Soll ein klimaresilienter und naturgemäßer Dauerwald erreicht werden, sind waldbauliche Maßnahmen erforderlich, die durch ein geeignetes Wildtiermanagement begleitet werden müssen. Waldverjüngung mit gleichzeitiger Vergrößerung der Baumartenvielfalt dient der Diversifizierung und Stabilisierung der Waldbestände, um gegenüber weiteren Klimaveränderungen resilienter zu werden und zugleich die Funktion als $\mathrm{CO}_{2}$-Senke dauerhaft zu erfüllen. Dies kann jedoch nur Erfolg haben, wenn es zugleich gelingt, den Äsungsdruck auf die Jungpflanzen durch flankierende Maßnahmen des Wildtiermanagements und der Besucherlenkung zu verringern. Das Anpassungsprojekt KLIMWALD 9 lief von 2015 bis zum Beginn des Jahres 2018 und hatte das Ziel, geeignete Methoden zur klimarobusten Entwicklung der Waldbestände und Lösungsansätze für bisherige Hürden der Klimaanpassung für die

\footnotetext{
9 https://www.umweltbundesamt.de/themen/klima-energie/ klimafolgen-anpassung/werkzeuge-der-anpassung/tatenbank/ klimwald-erfolgreiche-klimaanpassung-im (09.04.2021).
} 
Kommunalwälder im nordhessischen Habichtswald zu konzipieren (Godt/Henschke 2019: 7).

Notwendige Umsetzungsvoraussetzung für die Zielerreichung war die Kooperation der beteiligten relevanten Akteure. Als relevante Akteure standen die Eigentümer, der Forstbetrieb und die Jagdpächter im Fokus des Projektes. Um gemeinschaftlich getragene Lösungen für den Wald-WildKonflikt im Projektgebiet zu erarbeiten, wurde ein mehrschrittiger Prozess durchgeführt: In der ersten Phase wurde neues Wissen zu Klimawandel, Waldbau und Wildtiermanagement zusammengetragen (Kapazitätsbildung durch Vorträge und Exkursionen). Auf der Basis lokal differenzierter Analysen wurden die lokalen Anpassungsprobleme erfasst und mit den lokalen Wissensbeständen der Förster und Jäger vor Ort zusammengebracht.

Einvernehmlich wurden im zweiten Schritt Leitlinien der Kooperation verabredet, doch nach einem Jahr des Vertrauens- und Kapazitätsaufbaus stellte die wichtige Gruppierung der Jäger sich auf überörtlicher Ebene quer, Kreisund Landesjagdverband stiegen öffentlichkeitswirksam aus dem Prozess aus. Ziel der Aktion war die Sicherstellung der eigenen Belange, man wollte sich nicht die eigene Freiheit der Hegestrategie im Jagdrevier durch kollaborativ erarbeitete Regeln einschränken lassen. Die Exit-Option wurde gezogen, nachdem die Strategie des Bremsens nicht mehr erfolgreich erschien.

Die Absage an eine gemeinsame Systemsicht, das offene Verweigern transformativer Erkenntnisse wurde auch nach zwei Mediationsterminen nicht gebrochen. Das Scheitern des ersten Ansatzes und seine Reflexion brachten den Impuls zur Veränderung des Prozessdesigns (ganz im Sinne einer prozeduralen Planungstheorie; Allmendinger 2017: 192): Statt eine einheitliche Lösung für das gesamte regionale Wald-Wild-System anzustreben, wurde das Projektgebiet in kleinere Raumeinheiten aufgeteilt. Dazu wurden Teilregionen gebildet, um lokalspezifische Lösungen zu erarbeiten. Die verfahrene Gesamtsituation wurde aufgebrochen, indem neue Teilsituationen geschaffen und für diese Teilsituationen neue Diskurse entfaltet wurden. Dies veränderte zugleich die Struktur der Akteure, da nun die jeweiligen lokalen Vertreter/-innen und nicht mehr ihre überlokalen Repräsentanten an Lösungen vor Ort arbeiteten.

Dies funktionierte in einer Teilregion und dem in der dortigen Kollaboration entstandenen Handlungskonzept sehr gut (Hahne 2018: 98), in den Teilregionen mit ,negativer Koordination wird der Prozess mehr Zeit benötigen, aber das gute Beispiel hilft, die Chance für einen gemeinsamen Lernprozess aufrechtzuerhalten. Das Beispiel zeigt: Dialog bedeutet nicht, dass die Interessen und versteckten Agenden offengelegt werden. Auch das gut gemachte Prozessdesign führt nicht automatisch zu politischer Akzeptanz und ,das
Planungsergebnis ist noch lange nicht das Ergebnis und das Ende des Prozesses“" (Selle 2019: 8).

Das im Beispiel erzielte Ergebnis lässt sich daher auch kritisch interpretieren: Im Hinblick auf das Gesamtziel eines klimaangepassten Waldumbaus auf größerer Fläche wurden konsensuale Lösungsansätze nur für ein Teilgebiet erarbeitet. Das Gesamtziel wurde somit verfehlt. Das Änderungstempo reicht möglicherweise nicht aus, dem klimaänderungsbedingten Artensterben entgegenzuwirken. Das deliberative Verfahren kann, wie das Beispiel zeigt, als Katalysator den Transformationsprozess beschleunigen, aber eben auch verlangsamen, sodass vereinbarte politische Zielsetzungen nicht erreicht werden können. Im folgenden Kapitel werden die Erkenntnisse aus dem Beispiel genutzt, um Erweiterungen in den Theorien zu Kontingenz und Interventionen vorzuschlagen.

\section{Kontingenz und Interventionen in realweltlichen Prozessen}

Das obige Beispiel aus einem Klimaanpassungsprozess macht Unwägbarkeiten in Reallaboren anhand des Ausscherens wichtiger Akteure plastisch. Das Beispiel zeigt auch einen im veränderten Prozessdesign gewählten Weg, mit Überraschung umzugehen. Offenheit für Überraschungen ist daher eine ,wesentliche Anforderung an das Projektdesign wie an das Projektmanagement", wie Krohn, Grunwald und Ukowitz (2017: 344) fordern.

\subsection{Kontingenzen und Überraschungen als Elemente des Wandels}

Kontingenzen als gedachte Alternativentwicklungen und Überraschungen als plötzliche unerwartete Ereignisse bergen erhebliche Risiken und Chancen in Reallaboren (Karvonen/van Heur 2014: 388). Bei einem Verständnis von Reallaboren und städtischen (oder regionalen) Entwicklungsprozessen als lernenden Systemen bietet die Einbeziehung von Kontingenzen Möglichkeiten, sich auf Veränderungen einstellen zu können und andere Zukünfte zu denken.

Eine Überraschung ist - systemisch betrachtet - eine Störung in einem System, die entweder im System abgepuffert werden kann (Resilienz als Widerstand), das System verändert (Resilienz als Anpassung mit Neuorientierung des Systems) oder es gar zerstört (Zusammenbruch). Wann findet die Störung Beachtung? Ist es bereits die kleine Irritation, die in einem System zwar wahrgenommen wird, aber noch nicht genügend Informationssignale aussendet, um in den Rückkopplungen des Systems aufgenommen $\mathrm{zu}$ werden, oder erst das spätere systemverändernde Ergebnis? Ist es der Schneeball oder die Lawine, um auf 
Senge (1996: 106) zurückzugreifen? Überraschungen verändern Planungs- und Transformationsprozesse. Sie können negativer Art sein, wie die Beispiele zu destruktiven Akteurstrategien oben gezeigt haben, sie können aber auch als Prozessbeschleuniger dienen, indem sie zu neuen Lösungen treiben - das zwischenzeitliche Scheitern im obigen Beispiel als Anstoß zum Überdenken des Labordesigns oder Möglichkeiten eröffnen, die nicht durch das System selbst produziert werden können.

Kontingenzen und Überraschungen sind wesentliche Elemente der Praxisdynamik (und damit stets auch ein Einflussfaktor in Reallaboren): Seien es Naturkatastrophen, Lockdown-Effekte infolge einer Pandemie oder auch die positive Wendung in Prozessen beispielsweise durch einen neuen Akteur, der die erwünschte Pfadänderung herbeiführt. Viele Planerinnen und Planer kennen Momente der Unwägbarkeit in Prozessen: Da springen Investoren ab, auf deren Pläne das Konzept ausgerichtet war, da geht das Sprachrohr einer ganzen Gruppierung verloren, wenn ihr Sprecher fortzieht oder verstirbt, da werden Themen verknüpft, sodass der bisherige Prozess in neuem Licht erscheint und bislang unbehandelte Fragen aufwirft. Routiniers in Planung und Verwaltung reagieren dann damit, die „Überraschungen herunterzuspielen und rationale Planungsfähigkeiten zu demonstrieren“ (Hutter 2017: 158).

Überraschungen treten aber auch auf, wenn im Realexperiment bestimmte Hypothesen gebildet werden und die Abweichung von der Hypothese im Experiment eine produktive Überraschung darstellt (Groß 2017: 22). Überraschungen wirken somit als Katalysator und können Wandlungsprozesse verlangsamen oder beschleunigen. Kipppunkte im Prozess können also in unterschiedliche Richtungen deuten. Nicht immer ist dabei die Intuition ein guter Ratgeber, worauf Systemtheoretiker stets hinweisen (Meadows 1999: 1). Betrachten wir Überraschung als eine produktive Möglichkeit, festgefahrene und zähe Prozesse neu anzustoBen, so beginnt die Suche nach Instrumenten, für positive Überraschungen offen zu sein und Überraschungsmomente zu erzeugen. Bei der Öffnung für Überraschungen wird die Überraschungskompetenz geschult, also die Aufmerksamkeit für kleine Irritationen im System, welche später einmal zu Stress führen oder neue Perspektiven eröffnen könnten. Überraschungskompetenz und Kontingenzmanagement sind damit auch Beiträge, die Resilienz zu erhöhen. Überraschungsmomente zu kreieren und bewusst einzusetzen, ist ein Verfahren, dessen sich auch in Planungsprozessen bedient wird: Experimente und Interventionen stellen gute Möglichkeiten dar, Denkweisen infrage zu stellen, Blickwinkel zu verändern und neue Impulse in einen Prozess zu geben.

\subsection{Interventionen - gezielte Überraschungen}

Jedes Reallabor, jede Planung stellt eine Intervention in ein bestehendes System dar. Unter Interventionen werden hier kurzfristige, meist temporäre Eingriffe in Realsituationen verstanden, die nicht den Handlungsrahmen des Systems verändern, sondern aus einer aktiven Handlung im System bestehen und eine Veränderung der Situation bewirken. In diesem Sinne werden gezielte Interventionen als Veränderungsimpulse in Raumentwicklungsprozessen vielfach angewendet. Das Spektrum reicht von temporären technischen Interventionen in der Verkehrsplanung (vgl. obiges Beispiel der Verkehrsberuhigung) bis hin zu künstlerischen Praktiken. Es geht um das Sicht- und Fühlbarmachen von Veränderungen. So wird die Perspektive auf die Raumnutzung verändert, wenn eine Hauptverkehrsader für kurze Zeit Fußgängerinnen und Fußgängern gewidmet wird, wenn auf den Leerstand von Häusern hingewiesen wird durch künstlerische Gestaltung (Altrock/Beeck 2013: 21) oder durch Aktionen der Bewohner/-innen eine Wiederbelebung erfolgt (Matton 2017).

Die Methoden der gezielten Intervention im Kontext der Raumentwicklung reichen von dialogischen Verfahren über Bilder (Leitbilder, Raumbilder, Pläne; Obkircher 2017: 195), den Einsatz von „Change Agents“ (Kristof 2017: 165) oder künstlerische Eingriffe bis hin zu Experimenten. Der Begriff der Intervention gilt als unbestimmt und ambivalent (von Borries/Hiller/Kerber et al. 2012: 14). Gleichwohl kann er in Modelle der Transformation eingebaut werden. Weiterentwickelt wird dazu ein Modell von Ison (2016: 44), welches davon ausgeht, dass Praxistransformationen Veränderungen in zwei Richtungen veranlassen: einerseits auf der Ebene der Praxis, andererseits auf der Ebene des Verständnisses. Im Unterschied zum Modell von Ison wird hier die in Kapitel 4.3 benannte Ebene der mentalen Infrastrukturen (Überzeugungen, versteckte Agenden) hinzugefügt. Auf beiden Ebenen können Interventionen ansetzen (vgl. Abbildung 1).

Interventionen vom Typ 1 wirken direkt auf der Sachund Handlungsebene - mit allen Fragen zu Inhalt, Rahmenbedingungen, zu den Akteuren, den extern Betroffenen und ihren Interessen, zu Institutionen und Macht bis zu den Instrumenten. Eines der Instrumente auf dieser Ebene ist die Moderation des Kommunikationsprozesses. Interventionen vom Typ 2 zielen auf die Metaebene der mentalen Infrastrukturen und versuchen, verkrustete Strukturen, Denkweisen und Verfahren in den Veränderungsmodus zu versetzen und zu einem Verständniswandel anzuregen (Göpel 2016: 149). 


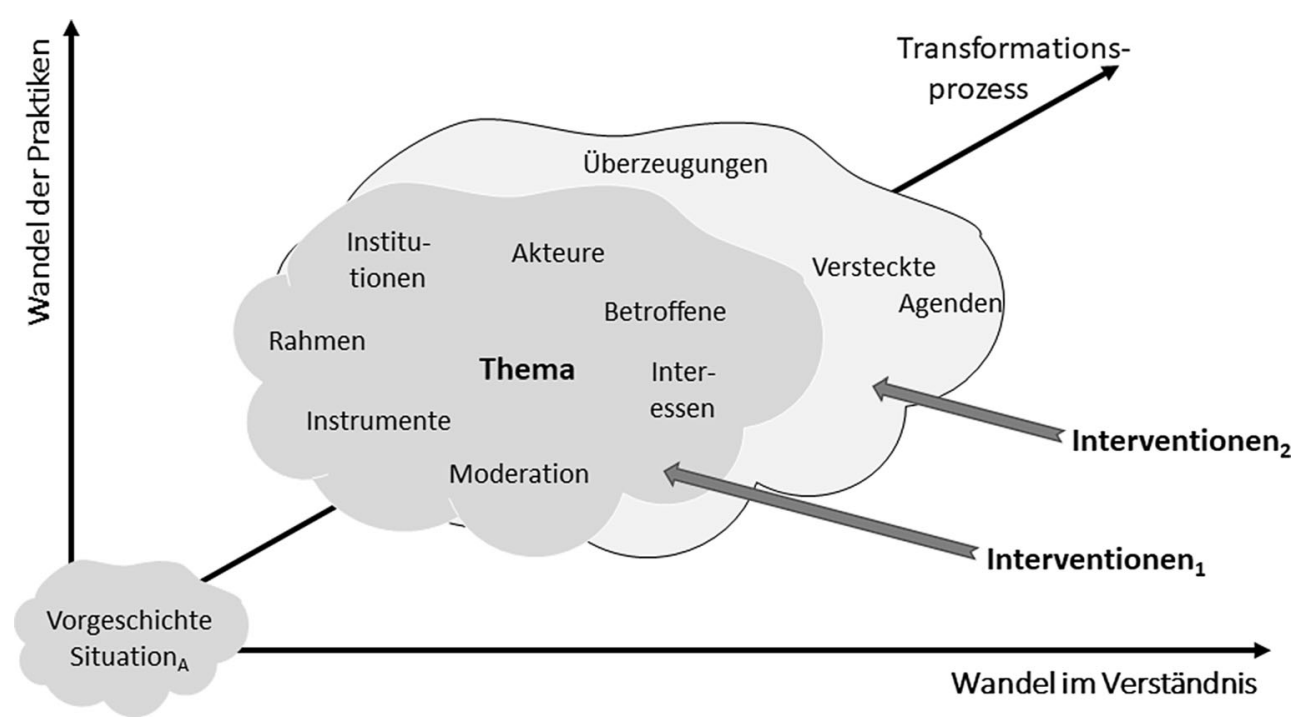

Abbildung 1 Interventionsebenen im Reallabor

Quelle: Eigene Darstellung in Anlehnung an Ison (2016: 44)

\subsection{Reallabore als Handlungsformat}

Mit ihrem „Interventionscharakter“ (WBGU 2016: 542) zielen Reallabore klar auf die Umsetzungsebene, es geht darum, Stadt „,mitzugestalten“ (WBGU 2016: 105). Gefordert werden „Ergebnisse in Form anwendungsfähiger Lösungen“ (WBGU 2016: 468). Viele Ansätze bewegen sich in genau den Feldern von Problemstellungen und Lösungsansätzen, welche auch in der Stadt- und Regionalentwicklung bearbeitet werden. Beispiele dafür finden sich bei Defila und Di Giulio (2019: 341-363), da geht es um typische Fälle aus der Verkehrsplanung (z. B. Verkehrsberuhigung, Stellplätze, Modal Split, autonomes Fahren), der Quartiersentwicklung bis hin zum Urban Gardening, der Energiewende vor Ort, der Entfaltung neuer Formen des Wirtschaftens. Damit wird auch auf der Handlungsebene die Frage nach dem Neuigkeitswert des Formats Reallabore aufgeworfen.

Neben problembezogenen Reallaboren, die an Sachthemen aufgezogen werden, finden sich auch offene Formate, in denen die Akteure selbst ihre Themen einbringen können, z. B. „Dein Nachhaltigkeitsexperiment“ beim „Reallabor 131: KIT findet Stadt“" (Meyer-Soylu/Parodi/Trenks et al. 2016: 37; Kanning 2018: 19). Auch hierfür finden sich in der forschenden Praxis der Stadtentwicklung Beispielformate, die seit Längerem eingesetzt werden wie Zukunftswerkstätten, Open Space oder World Cafés. Zu den zentralen Problemen der offenen Formate gehört die Entscheidungsfindung über die Priorisierung und Weiterverfolgung von Strategien und Projekten. Die Ansprüche der Bürger/-innen und des aktiven Personenkreises stoßen sich hier schnell mit den Realitäten von Politik, Verwaltung oder Projektverantwortlichen, wenn diese nicht bereit sind, mögliche neue
Ziele, alternative Wege oder die eigenen Prioritäten des Handelns infrage zu stellen (Selle 2019: 29). Da erscheint der Vorschlag, die beteiligten Akteure über die Präferierung der zu verfolgenden Szenarien entscheiden zu lassen (Lam/ Horcea-Milcu/Fischer et al. 2020: 1455), höchst fragwürdig. Gestaltungsoffenheit zu suggerieren, wenn Wunschkataloge längst bekannt, Themen in langen Schleifen anderer Prozesse diskutiert sind, die Umsetzung nicht gewollt oder nicht leistbar ist, gehört zu den Mechanismen, welche schon bei Lokale-Agenda-21-Prozessen zu Frustration und zum Rückzug vieler Akteure beigetragen haben (Hahne 2002: 28).

\section{Resümee}

Neue Begriffe stimulieren produktive Auseinandersetzungen. Dies ist auch mit dem von den transformativen Wissenschaften eingeführten Vorschlag verbunden, Reallabore als Forschungs- und Beteiligungsformat zur Überwindung von Blockaden und Handlungsdefiziten der Nachhaltigkeitstransformation einzuführen. Der Ansatz der Reallabore schlägt vor, mittels eines transdisziplinären und beteiligungsorientierten Vorgehens umsetzungsrelevante Akteure zu gewinnen und mit ihnen gemeinsam im Bearbeitungsprozess Wissenslücken zu verringern. Neue Formate können dabei neues Interesse und neubelebte Motivation, neue Konstellationen der Kollaboration und neues Transformationswissen erzeugen. Daher ist es nicht verwunderlich, dass dieser Begriff rasch Eingang in Forschung und Praxis der Stadtund Regionalentwicklung gefunden hat und die Nachhal- 
tigkeitsanstrengungen vor Ort mit einem neuen Format unterstützt.

Der Beitrag sollte zeigen, dass es in der Raumentwicklung langjährige Erfahrungen mit Beteiligungs- und Kollaborationsprozessen gibt, auf welche eine transformative Wissenschaft zurückgreifen kann. Die Fehler und Fallstricke, die bei Prozessdesign, Akteurauswahl, Beteiligungsverfahren, Rückkopplungsschleifen, Lernprozessen und Umsetzungsverfahren gemacht werden können, sind gut beschrieben und erlauben es, heute mit deutlich weniger „Naivität“ (Selle 2019) und größerer ortsspezifischer Sensibilität im Feld der transdisziplinären Forschung und Planung voranzugehen, Beteiligungsformate und Interventionen gezielt und reflektiert zu setzen, Scheitern zum Lernen und Interventionen für kreative Anregungen zu nutzen.

\section{Literatur}

Albiez, M.; Meyer-Soylu, S.; Parodi, O.; Waitz, C. (2016):

Das „Quartier Zukunft - Labor Stadt“: ein reales Reallabor. In: Hahne, U.; Kegler, H. (Hrsg.): Resilienz. Stadt und Region - Reallabore der resilienzorientierten Transformation. Frankfurt am Main, 101125. = Stadtentwicklung 1. https://doi.org/10.3726/978-3-653-06657-9

Allmendinger, P. (2017): Planning Theory. London.

Altrock, U.; Beeck, S. (2013): Stadtentwicklung und die Praxis von „Interventionen“. In: Raumplanung 167, 2, 21-26.

ARL - Akademie für Raumforschung und Landesplanung (2000): Nachhaltigkeitsprinzip in der Regionalplanung. Handreichung zur Operationalisierung. Hannover. = Forschungs- und Sitzungsberichte 212.

Below, N.; Dopfer, J. (2011): Stadttypische Formen der Bürgerbeteiligung: Eine Annäherung an Frankfurt und München. In: Löw, M.; Terizakis, G. (Hrsg.): Städte und ihre Eigenlogik. Ein Handbuch für Stadtplanung und Stadtentwicklung. Frankfurt am Main, 225-235. = Interdisziplinäre Stadtforschung 11.

Berking, H.; Löw, M. (Hrsg.) (2008): Die Eigenlogik der Städte. Neue Wege für die Stadtforschung. Frankfurt am Main. = Interdisziplinäre Stadtforschung 1.

Bischoff, A.; Selle, K.; Sinning, H. (2005): Informieren, Beteiligen, Kooperieren: Kommunikation in Planungsprozessen. Dortmund.

BMBF - Bundesministerium für Bildung und Forschung (2015): Zukunftsstadt. Strategische Forschungs- und Innovationsagenda. Berlin.

BMWi - Bundesministerium für Wirtschaft und Energie (2019): Freiräume für Innovation. Das Handbuch für Reallabore. Berlin.

Borner, J.; Kraft, A. H. (2018): Konzeptpapier zur Reallabor-Methode im ENavi-Projekt. http://kmgne.de/
wp-content/uploads/2019/04/ENavi_AP13_KMGNE_ Konzeptpapier_Reallabore_Borner-Kraft_Jan2018.pdf (25.02.2021).

Brand, K.-W.; Christ, E.; Heimerl, A.; Rau, A.; Warsewa, G. (2001): Bedingungen institutioneller Stabilisierung lokaler Agenda 21-Prozesse. München.

Bulkeley, H.; Betsill, M. (2005): Rethinking Sustainable Cities: Multilevel Governance and the 'Urban' Politics of Climate Change. In: Environmental Politics 14, 1, 4263. doi.org/10.1080/0964401042000310178

Bulkeley, H.; Castán Broto, V. (2012): Government by Experiment? Global Cities and the Governing of Climate Change. In: Transactions of the Institute of British Geographers 38, 3, 361-375. https://doi.org/10.1111/j.14755661.2012.00535.x

Bulkeley, H.; Marvin, S.; Palgan, Y. V.; McCormick, K.; Breitfuss-Loidl, M.; Mai, L.; von Wirth, T.; Frantzeskaki, N. (2019): Urban Living Laboratories: Conducting the Experimental City? In: European Urban and Regional Studies 26, 4, 317-335. https://doi.org/10.1177/ 0969776418787222

Callon, M.; Lascoumes, P.; Barthe, Y. (2009): Acting in an Uncertain World. An Essay on Technical Democracy. Cambridge/Massachusetts.

Defila, R.; Di Giulio, A. (2018): Reallabore als Quelle für die Methodik transdisziplinären und transformativen Forschens - eine Einführung. In: Defila, R.; Di Giulio, A. (Hrsg.): Transdisziplinär und transformativ forschen. Eine Methodensammlung. Wiesbaden, 9-35. https://doi. org/10.1007/978-3-658-21530-9_1

Defila, R.; Di Giulio, A. (Hrsg.) (2019): Transdisziplinär und transformativ forschen. Band 2. Eine Methodensammlung. Wiesbaden. https://doi.org/10.1007/978-3658-27135-0

De Flander, K.; Hahne, U.; Kegler, H.; Lang, D.; Lucas, R.; Schneidewind, U.; Simon, K.-H.; Singer-Brodowski, M.; Wanner, M.; Wiek, A. (2014): Resilience and Real-life Laboratories as Key Concepts for Urban Transition Research. In: GAIA - Ecological Perspectives for Science and Society 23, 3, 284-286. https://doi.org/10. 14512/gaia.23.3.19

Di Giulio, A.; Defila, R.; Brückmann, T. (2016): „Das ist halt das eine ... Praxis, das andere ist Theorie“. Prinzipien transdisziplinärer Zusammenarbeit im Forschungsalltag. In: Defila, R.; Di Giulio, A. (Hrsg.): Transdisziplinär forschen - zwischen Ideal und gelebter Praxis. Hotspots, Geschichten, Wirkungen. Frankfurt am Main, 189-286.

Engels, A.; Walz, K. (2018): Dealing with Multi-Perspectivity in Real-World Laboratories. Experiences from the Transdisciplinary Research Project Urban Transformation Laboratories. In: GAIA - Ecological Perspectives for 
Science and Society 27, S1, 39-45. https://doi.org/10. 14512/gaia.27.S1.10

Engels, F.; Rogge, C.-J. (2018): Tensions and Trade-offs in Real-World Laboratories - The Participants' Perspective. In: GAIA - Ecological Perspectives for Science and Society 27, S1, 28-31. https://doi.org/10.14512/gaia.27.S1. 8

Evans, J.; Karvonen, A. (2014): „Give Me a Laboratory and I Will Lower Your Carbon Footprint!“ Urban Laboratories and the Governance of Low-Carbon Futures. In: International Journal of Urban and Regional Research 38, 2, 413-430. https://doi.org/10.1111/1468-2427.12077

Geels, F. W. (2011): The multi-level perspective on sustainability transitions: Responses to seven criticisms. In: Environmental Innovation and Societal Transitions 1, 1, 24-40. https://doi.org/10.1016/j.eist.2011.02.002

Gerhard, U.; Marquardt, E. (2017): Reallabore als innovatives Forschungsformat zur Untersuchung nachhaltiger Stadtentwicklung - eine kritische Reflexion. In: Berichte. Geographie und Landeskunde 91, 1, 97-111.

Gerhard, U.; Marquardt, E.; West, C. (2017): Reallabore in der Stadtforschung. Eine Einführung. In: Berichte. Geographie und Landeskunde 91, 1, 5-12.

Godt, J.; Henschke, C. (2019): Konzeptionelle und methodische Aspekte für die Erarbeitung von Wildtiermanagementplänen. Kassel.

Göpel, M. (2016): The Great Mindshift. How a New Economic Paradigm and Sustainability Transformations go Hand in Hand. Cham. https://doi.org/10.1007/978-3319-43766-8

Gothe, S. (2006): Regionale Prozesse gestalten. Handbuch für Regionalmanagement und Regionalberatung. Kassel.

Groß, M. (2017): Experimentelle Kultur und die Governance des Nichtwissens. In: Reinermann, J.-L.; Behr, F. (Hrsg.): Die Experimentalstadt. Kreativität und die kulturelle Dimension der Nachhaltigen Entwicklung. Wiesbaden, 21-40. https://doi.org/10.1007/978-3-65814981-9_2

Groß, M.; Hoffmann-Riem, H.; Krohn, W. (2005): Realexperimente. Ökologische Gestaltungsprozesse in der Wissensgesellschaft. Bielefeld. https://doi.org/10.14361/ 9783839403044

Hahne, U. (2002): Lokale Agenda 21 als Basis nachhaltiger Regionalentwicklung - Dilemmata eines neuen Politiktypus. In: Geographische Revue 2, 21-33.

Hahne, U. (2008): ELER und der unterschiedliche Mehrwert des LEADER-Ansatzes in Deutschland. In: Bundesministerium für Ernährung, Landwirtschaft und Verbraucherschutz (Hrsg.): Zukunft ländlicher Räume. Stuttgart, 127-134. = Berichte über Landwirtschaft, Sonderheft 217.

Hahne, U. (2018): Dilemmata, Blockaden und Fallstricke in
Reallaboren. Ein Beitrag zur kommunikativen Planung anhand eines Klimaanpassungsvorhabens im Wald. In: Hennecke, S.; Kegler, H.; Klaczynski, K.; Münderlein, D. (Hrsg.): Diedrich Bruns wird gelehrt haben. Eine Festschrift. Kassel, 93-102.

Holling, C. S.; Gunderson, L. H. (2002): Resilience and Adaptive Cycles. In: Gunderson, L. H.; Holling, C. S. (Hrsg.): Panarchy. Understanding Transformations in $\mathrm{Hu}-$ man and Natural Systems. Washington, 25-62.

Hutter, G. (2017): Dealing with Surprise in Urban Regions. Some Ideas and Examples for Planners. In: Deppisch, S. (Hrsg.): Urban Regions Now \& Tomorrow. Between Vulnerability, Resilience and Transformation. Wiesbaden, 14516-4. https://doi.org/10.1007/978-3-658-16759-2_7

Ison, R. (2016): What is Systemic about Innovation Systems? The Implications for Policies, Governance and Institutionalization. In: Francis, J.; Mytelka, L.; van Huis, A.; Röling, N. (Hrsg.): Innovation systems: towards effective strategies in support of smallholder farmers. Wageningen, 37-52.

Jakubowski, P. (2016): Resiliente Stadtentwicklung - Modellvorhaben können helfen. In: Hahne, U.; Kegler, H. (Hrsg.): Resilienz. Stadt und Region - Reallabore der resilienzorientierten Transformation. Frankfurt am Main, 147-165. = Stadtentwicklung 1 .

Janich, P. (2004): Experiment. In: Mittelstraß, J. (Hrsg.): Enzyklopädie Philosophie und Wissenschaftstheorie. Stuttgart, 621-622.

Kanning, H. (2018): Reallabore aus planerischer Perspektive. Hannover. $=$ sustainify Arbeits- und Diskussionspapier 3.

Karvonen, A.; van Heur, B. (2014): Urban Laboratories: Experiments in Reworking Cities. In: International Journal of Urban and Regional Research 38, 2, 379-392. https:// doi.org/10.1111/1468-2427.12075

Kristof, K. (2017): Change Agents in gesellschaftlichen Veränderungsprozessen. In: Reinermann, J.-L.; Behr, F. (Hrsg.): Die Experimentalstadt. Kreativität und die kulturelle Dimension der Nachhaltigen Entwicklung. Wiesbaden, 165-179. https://doi.org/10.1007/978-3658-14981-9_9

Krohn, W.; Grunwald, A.; Ukowitz, M. (2017): Transdisziplinäre Forschung revisited. Erkenntnisinteresse, Forschungsgegenstände, Wissensform und Methodologie. In: GAIA - Ecological Perspectives for Science and Society 26, 4, 341-347. https://doi.org/10.14512/gaia. 26.4.11

Kullman, K. (2013): Geographies of Experiment/Experimental Geographies. A Rough Guide. In: Geography Compass 7, 12, 879-894. https://doi.org/10.1111/gec3. 12087

Lam, D. P. M.; Horcea-Milcu, A. I.; Fischer, J.; Peukert, 
D.; Lang, D. J. (2020): Three principles for co-designing sustainability intervention strategies: Experiences from Southern Transylvania. In: Ambio 49, 1451-1465. https://doi.org/10.1007/s13280-019-01302-x

Leminen, S.; DeFillippi, R.; Westerlund, M. (2015): Paradoxical Tensions in Living Labs. In: Huizingh, E.; Torkkeli, M.; Conn, S.; Bitran, I. (Hrsg.): Proceedings of the XXVI ISPIM Conference. Manchester, 1-14.

Löw, M. (2011): Lokale Ökonomie - Lebensqualität als Standortfaktor. In: Löw, M.; Terizakis, G. (Hrsg.): Städte und ihre Eigenlogik. Ein Handbuch für Stadtplanung und Stadtentwicklung. Frankfurt am Main, 29-35. = Interdisziplinäre Stadtforschung 11.

Marvin, S.; Bulkeley, H.; Mai, L.; McCormick, K.; Palgan, Y. V. (Hrsg.) (2018): Urban Living Labs. Experimenting with City Futures. Abingdon.

Matton, T. (Hrsg.) (2017): Dorf machen. Improvisationen zur sozialen Wiederbelebung. Berlin.

McCormick, K.; Hartmann, C. (2017): The Emerging Landscape of Urban Living Labs. Characteristics, Practices and Examples. Lund. https://lup.lub.lu.se/search/ ws/files/27224276/Urban_Living_Labs_Handbook.pdf (25.02.2021).

McCormick, K.; Anderberg, S.; Coenen, L.; Neij, L. (2013): Advancing Sustainable Urban Transformation. In: Journal of Cleaner Production 50, 1-11. https://doi.org/10. 1016/j.jclepro.2013.01.003

Meadows, D. (1999): Leverage Points. Places to Intervene in a System. Hartland.

Meyer-Soylu, S.; Parodi, O.; Trenks, H.; Seebacher, A. (2016): Das Reallabor als Partizipationskontinuum. Erfahrungen aus dem Quartier Zukunft und Reallabor 131 in Karlsruhe. In: Technikfolgenabschätzung - Theorie und Praxis 25, 3, 31-40. https://doi.org/10.14512/tatup. 25.3.31

MWFK - Ministerium für Wissenschaft, Forschung und Kunst Baden-Württemberg (2013): Wissenschaft für Nachhaltigkeit. Herausforderung und Chance für das baden-württembergische Wissenschaftssystem. Stuttgart.

Obkircher, S. (2017): Raumentwicklung in Grenzregionen. Bedeutung und Wirkung von Planungsleitbildern und Governance-Prozessen. Bielefeld. = Sozial- und Kulturgeographie 15 .

Overdevest, C.; Bleicher, A.; Gross, M. (2010): The experimental turn in environmental sociology: Pragmatism and new forms of governance. In: Gross, M.; Heinrichs, H. (Hrsg.): Environmental Sociology. Dordrecht, 279-294. https://doi.org/10.1007/978-90-481-8730-0_16

Park, R. E.; Burgess, E. W. (1984): The City. Suggestions for Investigation of Human Behavior in the Urban Environment. Reprint (Original 1925). Chicago.
Parodi, O.; Ley, A.; Fokdal, J.; Seebacher, A. (2018): Empfehlungen für die Förderung und den Aufbau von Reallaboren. Ein Positionspapier der BaWü-Labs. https:// www.quartierzukunft.de/Positionspapier-BaWue-Labs. pdf (22.01.2021).

Parodi, O.; Waitz, C.; Bachinger, M.; Kuhn, R.; Meyer-Soylu, S.; Alcántara, S.; Rhodius, R. (2018): Insights into and Recommendations from Three Real-World Laboratories. An Experience-Based Comparison. In: GAIA Ecological Perspectives for Science and Society 27, S1, 52-59. https://doi.org/10.14512/gaia.27.S1.12

Rittel, H. W. J.; Webber, M. M. (1973): Dilemmas in a General Theory of Planning. In: Policy Sciences 4, 155-169. https://doi.org/10.1007/BF01405730

Rogga, S.; Zscheischler, J.; Gaasch, N. (2018): How Much of the Real-World Laboratory Is Hidden in Current Transdisciplinary Research? In: GAIA - Ecological Perspectives for Science and Society 27, S1, 18-22. https://doi. org/10.14512/gaia.27.S1.6

Rose, M.; Wanner, M.; Hilger, A. (2019): Das Reallabor als Forschungsprozess und -infrastruktur für nachhaltige Entwicklung. Konzepte, Herausforderungen und Empfehlungen. o.O. = NaWiKo Synthese Working Paper 1 .

Schäpke, N.; Stelzer, F.; Bergmann, M.; Singer-Brodowski, M.; Wanner, M.; Caniglia, G.; Lang, D. J. (2017): Reallabore im Kontext transformativer Forschung. Ansatzpunkte zur Konzeption und Einbettung in den internationalen Forschungsstand. Lüneburg. = IETSR Discussion Papers in Transdisciplinary Sustainability Research 1/2017.

Schäpke, N.; Stelzer, F.; Caniglia, G.; Bergmann, M.; Wanner, M.; Singer-Brodowski, M.; Loorbach, D.; Olsson, P.; Baedeker, C.; Lang, D. J. (2018): Jointly Experimenting for Transformation? Shaping Real-World Laboratories by Comparing Them. In: GAIA - Ecological Perspectives for Science and Society 27, S1, 85-96. https://doi.org/ 10.14512/gaia.27.S1.16

Schneidewind, U. (2014): Urbane Reallabore - ein Blick in die aktuelle Forschungswerkstatt. In: PND Online 3, $1-7$.

Schneidewind, U.; Singer-Brodowski, M. (2014): Transformative Wissenschaft: Klimawandel im deutschen Wissenschafts- und Hochschulsystem. Marburg.

Seebacher, A.; Alcántara, S.; Quint, A. (2018): Der Partizipationsmythos „Partizipation bedeutet, alle immer an allem zu beteiligen“. In: Defila, R.; Di Giulio, A. (Hrsg): Transdisziplinär und transformativ forschen. Eine Methodensammlung. Wiesbaden, 101-104. https://doi.org/10. 1007/978-3-658-21530-9_5

Selle, K. (2006): Shut down. Restart ... Vorschläge zur Wiederaufnahme der Diskussion über die Entwicklung von Städten und Regionen und den möglichen Beitrag öffentlicher Akteure. In: Selle, K. (Hrsg.): Planung neu denken. 
Band 2: Praxis der Stadt- und Regionalentwicklung. Analysen. Erfahrungen. Folgerungen. Dortmund, 557-577.

Selle, K. (2019): Ende der Naivität? Öffentlichkeitsbeteiligung in der Stadtentwicklung. Anstiftungen zur Revision. Berlin. $=$ Vhw-Schriftenreihe 15 .

Senge, P. M. (1996): Die fünfte Disziplin. Kunst und Praxis der lernenden Organisation. Stuttgart.

Seuberlich, M. (2012): Interkommunale Zusammenarbeit als Rettungsanker? Wie die Länder dieses Instrument für ihre Kommunen nutzen. In: dms - der moderne staat Zeitschrift für Public Policy, Recht und Management 5, 1, 105-124.

Siedentop, S.; Zimmer-Hegmann, R. (2020): Wahrheitssuche, realweltliche Problemlösungen und Autonomie. Erkundungen an der Schnittstelle zwischen Wissenschaft und Politik. In: Informationen zur Raumentwicklung 2, 26-37.

Singer-Brodowski, M.; Beecroft, R.; Parodi, O. (2018): Learning in Real-World Laboratories. A Systematic Impulse for Discussion. In: GAIA - Ecological Perspectives for Science and Society 27, S1, 23-27. https://doi.org/ 10.14512/gaia.27.S1.7

Ther, P. (2014): Die neue Ordnung auf dem alten Kontinent. Eine Geschichte des neoliberalen Europa. Berlin.

von Borries, F.; Hiller, C.; Kerber, D.; Wegner, F.; Wenzel, A.-L. (2012): Glossar der Interventionen: Annäherung an einen überverwendeten, aber unterbestimmten Begriff. Berlin.

Voytenko, Y.; McCormick, K.; Evans, J.; Schliwa, G. (2016): Urban living labs for sustainability and low carbon cities in Europe: Towards a research agenda. In: Journal of Cleaner Production 123, 45-54. https://doi. org/10.1016/j.jclepro.2015.08.053

Wagner, F.; Grunwald, A. (2015): Reallabore als Forschungs- und Transformationsinstrument: Die Quadratur des Hermeneutischen Zirkels. In: GAIA - Ecological Perspectives for Science and Society 24, 1, 26-31. https:// doi.org/10.14512/gaia.24.1.7

Wagner, F.; Grunwald, A. (2019): Reallabore zwischen Be- liebtheit und Beliebigkeit. Eine Bestandsaufnahme des transformativen Formats. In: GAIA - Ecological Perspectives for Science and Society 28, 3, 260-264. https://doi. org/10.14512/gaia.28.3.5

Walker, B. H.; Salt, D. (2006): Resilience Thinking. Sustaining Ecosystems and People in a Changing World. Washington.

Wanner, M.; Stelzer, F. (2019): Reallabore - Perspektiven für ein Forschungsformat im Aufwind. Wuppertal. = inbrief 7/2019.

Wanner, M.; Hilger, A.; Westerkowski, J.; Rose, M.; Stelzer, F.; Schäpke, N. (2018): Towards a Cyclical Concept of Real-World Laboratories. A Transdisciplinary Research Practice for Sustainability Transitions. In: disP - The Planning Review 54, 2, 94-114. https://doi.org/10.1080/ 02513625.2018.1487651

WBGU - Wissenschaftlicher Beirat der Bundesregierung Globale Umweltveränderungen (2011): Welt im Wandel Gesellschaftsvertrag für eine Große Transformation. Berlin.

WBGU - Wissenschaftlicher Beirat der Bundesregierung Globale Umweltveränderungen (2016): Der Umzug der Menschheit: Die transformative Kraft der Städte. Berlin.

Wékel, J. (2011): Lokale Planungskulturen - Zur Eigenlogik Münchens und Frankfurts. In: Löw, M.; Terizakis, G. (Hrsg.): Städte und ihre Eigenlogik. Ein Handbuch für Stadtplanung und Stadtentwicklung. Frankfurt am Main, 215-224. = Interdisziplinäre Stadtforschung 11.

Welzer, H. (2011): Mentale Infrastrukturen. Wie das Wachstum in die Welt und in die Seelen kam. Berlin. = Schriftenreihe Ökologie 14.

Wiechmann, T.; Mörl, K.; Vock, A. (2012): Evaluation von Modellvorhaben der Raumordnung. In: Informationen zur Raumentwicklung Heft 1/2, 79-90.

Zimmermann, K.; Feiertag, P. (2020): Zwischen und Theorie und Praxis. Die Rolle der wissenschaftlichen Politikberatung. In: Informationen zur Raumentwicklung 2, 3847. 